Jan Menke

Jörg Larsen

\section{Contrast-enhanced magnetic resonance angiography in carotid artery disease: does automated image registration improve image quality?}

Received: 14 August 2008

Accepted: 6 November 2008

Published online: 10 January 2009

(C) The Author(s) 2008.

This article is published with open access at Springerlink.com

\section{Electronic supplementary material The} online version of this article (doi:10.1007/ s00330-008-1257-6) contains supplementary material, which is available to authorized users.

\author{
J. Menke $(\bowtie)$ \\ Department of Diagnostic Radiology, \\ University Hospital, \\ Robert Koch Strasse 40, \\ 37075 Goettingen, Germany \\ e-mail: Menke-J@T-Online.de \\ Tel.: +49-551-5053614 \\ Fax: +49-551-5053614

\section{J. Larsen} \\ Institute for Roentgendiagnostics, \\ Braunschweig Teaching Hospitals, \\ Freisestrasse 9-10, \\ 38118 Braunschweig, Germany
}

\begin{abstract}
Contrast-enhanced magnetic resonance angiography (MRA) is a noninvasive imaging alternative to digital subtraction angiography (DSA) for patients with carotid artery disease. In DSA, image quality can be improved by shifting the mask image if the patient has moved during angiography. This study investigated whether such image registration may also help to improve the image quality of carotid MRA. Data from 370 carotid MRA examinations of patients likely to have carotid artery disease were prospectively collected. The standard nonregistered MRAs were compared to automatically linear, affine and warp registered MRA by using three image quality parameters: the vessel detection probability (VDP) in maximum intensity projection (MIP) images, contrast-to-noise ratio (CNR) in MIP images, and contrast-to-noise ratio in three-dimensional image vol-
\end{abstract}

umes. A body shift of less than $1 \mathrm{~mm}$ occurred in $96.2 \%$ of cases. Analysis of variance revealed no significant influence of image registration and body shift on image quality $(p>0.05)$. In conclusion, standard contrastenhanced carotid MRA usually requires no image registration to improve image quality and is generally robust against any naturally occurring body shift.

Keywords Carotid artery disease . Carotid stenosis - Magnetic resonance angiography $\cdot$ Automated image registration
Abbreviations CNR-3D: contrast- to-noise ratio (CNR) of three- dimensional (3D) images · CNR-MIP: CNR of angiographic maximum intensity projection (MIP) images . VDP: vessel detection probability

\section{Introduction}

Atherosclerotic extracranial carotid stenosis has a high prevalence in the elderly and is a treatable risk factor for ischaemic stroke, causing approximately $20 \%$ of cases [1-4]. Duplex ultrasonography is the primary imaging investigation in the assessment of carotid stenosis, whereas catheter-based digital subtraction angiography (DSA) represents the gold standard [5]. For solely diagnostic purposes, DSA is increasingly being replaced by noninvasive imaging alternatives, which include computed tomographic angiography (CTA) and contrast-enhanced magnetic resonance angiography (MRA) [5-7].
Angiographic image quality in DSA can be improved by digitally moving the mask image in cases when a patient has moved slightly during the image acquisition. This socalled image registration intends to eliminate the effect of the patient's body shift, such that the final subtracted DSA images are ideally free of motion artefacts. The quality of carotid subtraction CTA can also be increased by image registration with the intention to eliminate high-density structures such as bones, calcified plaques or implanted stent material from the final angiographic images [8-10]. Furthermore, the angiographic quality of lower leg MRA may be improved by image registration if the patient has moved their legs by $1 \mathrm{~mm}$ or more between acquisitions of 
the unenhanced and contrast-enhanced sequences [11]. The purpose of this study was to investigate whether such image registration could also improve the image quality in contrast-enhanced carotid MRA.

\section{Materials and methods}

\section{Patients}

Data from 375 carotid MRA examinations of patients with known or suspected carotid stenosis were prospectively collected over a period of 4 years. Informed patient consent had been obtained before MRA. The local institutional review board waived ethical approval of this study on image quality. MRA with sequences different from that described below were not included. Five MRAs were excluded from further analysis: one with insufficient arterial contrast, two with excessive venous contrast and two with technical artefacts. The remaining 370 MRA examinations had been obtained in 343 patients, including 27 follow-up MRAs. These follow-up MRAs were not excluded to prevent a selection bias, when transferring the study results to a similar MRA collective, i.e. an MRA collective that also includes follow-up MRA. Patient age ranged from 42 to 89 years (median 69) and body weight from 47 to $170 \mathrm{~kg}$ (median 79); the patient sample thus comprised a broad range of patient age and body size. The female-to-male gender ratio was 1:2.

\section{Magnetic resonance angiography}

The cervical MRAs were performed on clinical 1.5-T magnet systems (Magnetom Symphony and Sonata, Siemens, Erlangen, Germany) with standard head and neck receiver coils. Patients were placed headfirst in the magnet and were instructed to keep still. The need to reduce body motion was facilitated by gentle head fixation. Additionally the patients were advised not to breathe or swallow during image acquisition. The arterial circulation time was measured by using a 2-mL gadopentetate dimeglumine test bolus (Gd-DPTA, Magnevist, Schering, Germany). This bolus was injected into an antecubital vein and flushed with $25 \mathrm{~mL}$ physiological saline at a constant rate of $3 \mathrm{~mL} / \mathrm{s}$ using an MR injector (Spectris Medrad, Indianola, PA, USA). Thereafter, the supra-aortic arteries were imaged from the aortic arch to the circle of Willis by using a threedimensional (3D) gradient-echo sequence, resulting in 72 consecutive coronal slices (FLASH 3D, TR $3.54 \mathrm{~ms}$, TE $1.39 \mathrm{~ms}$, flip angle $25^{\circ}$, FOV $320 \times 208$ pixels, isotropic voxel size $1.1 \times 1.1 \times 1.1 \mathrm{~mm}$, acquisition time $21 \mathrm{~s}$ ). This $3 \mathrm{D}$ sequence was applied with sequential $k$-space ordering and multidimensional $6 / 8$ partial Fourier acquisitions in both the phase- and slice-encoding directions. First, such a 3D sequence was measured unenhanced. Then $0.2 \mathrm{~mL} / \mathrm{kg}$
Gd-DPTA was injected intravenously and flushed with $25 \mathrm{~mL}$ saline at a rate of $3 \mathrm{~mL} / \mathrm{s}$. The subsequent bolustimed gadolinium-enhanced 3D sequence was started such that central $k$-space acquisition coincided with the estimated arterial time-to-peak. The unenhanced 3D sequence was then subtracted from the gadoliniumenhanced 3D sequence to enhance the vascular contrast and suppress surrounding neck tissues [12]. From these 3D subtraction images, targeted maximum intensity projections (MIPs) were derived to depict the neck arteries [13]. Diagnostic radiological evaluation was based on these maximum intensity projections and on the original MRA images.

Postprocessing with automated image registration

The original DICOM images were converted to threedimensional ANALYZE image volumes by using the freeware program MRIcro (Rorden C, MRIcro version 1.40, http://www.sph.sc.edu/comd/rorden/mricro.html). The unenhanced image volume was then subtracted from the contrast-enhanced image volume to enhance the arteries and to reduce signals from the surrounding tissue. Angiographic maximum intensity projection (MIP) images were then generated at 12 different view angles in steps of $15^{\circ}$ to simulate a rotational projection angiography. The resulting images are standard MRA images without image registration. Additionally automated image registration with linear, affine and warp registration was applied to optimally fit the unenhanced image volume to its contrastenhanced counterpart $[14,15]$. This was done with the freeware automated image registration software package AIR 5.2.5 (Woods RP, AIR 5.2.5, http://bishopw.loni.ucla. edu/AIR5/). The registration time for automated image registration refers to calculation on a personal computer with a 2.4-GHz Pentium Quad processor and 1.5-GB working memory. Further postprocessing details are given in the Electronic supplementary material.

\section{Body shift}

Body shift between the unenhanced and contrast-enhanced 3D images was estimated without user interaction from the automated linear registration results by averaging the calculated three-dimensional shift of all tissue voxels in the corresponding organ region. This body shift was stratified into five shift classes from $0 \mathrm{~mm}$ to $\geq 4 \mathrm{~mm}$ in steps of $1 \mathrm{~mm}$.

\section{Quantitative computer-assisted evaluation}

The MRA image quality was assessed by computerassisted evaluation, which results in identical findings when repeated. In each single evaluation the automated 
analysis was blinded regarding the patient details and the results of the other evaluations. With reference to Sun and Parker, three image quality parameters were calculated [13]: (a) the vessel detection probability of an MIP image (VDP), (b) the contrast-to-noise ratio of an MIP image (CNR-MIP), and (c) the contrast-to-noise ratio of a 3D subtraction image (CNR-3D). For any MIP image, VDP quantifies the fraction of the vessels that can be detected within their surrounding tissue. The surrounding tissue was defined as the region at a distance of up to $5 \mathrm{~mm}$ away from the vessels. For any MIP image, CNR-MIP quantifies the ratio of vessel contrast (mean vessel intensity minus mean surrounding tissue intensity) to background noise (standard deviation of surrounding tissue intensities). For any 3D subtraction image, CNR-3D quantifies a similar ratio. The influence of body shift and registration method on the three image quality parameters was studied by two-factor analysis of variance (ANOVA) for the whole study group $[16,17]$. A further subgroup ANOVA was added for cases with $1-\mathrm{mm}$ or greater body shift to exclude any statistical influence from the cases with less than 1-mm body shift.

\section{Statistics}

Statistical analyses were performed using Statgraphics Plus for Windows 2.1 (1994, Manugistics, Rockville, Maryland, USA). The two-sided significance level was set to $p<0.05$.

\section{Results}

\section{Registration time}

In the included 370 MRAs the average $( \pm \mathrm{SD})$ registration time was $53( \pm 37) \mathrm{s}$ for linear registration, $124( \pm 118) \mathrm{s}$ for affine registration and $13( \pm 4)$ min for warp registration.

\section{Body shift}

For 356 out of 370 cases (96.2\%) a body shift of less than $1 \mathrm{~mm}$ occurred between acquisition of the unenhanced and the contrast-enhanced MR sequences. A body shift of at least $1 \mathrm{~mm}$ (shift class $\geq 2$ ) was found in 14 cases $(3.8 \%)$. Only five cases (1.4\%) exhibited a body shift of $2 \mathrm{~mm}$ or more.

Quantitative computer-assisted evaluation of MRA image quality

In almost all cases, VDP was already nearly $100 \%$ without image registration, indicating that the cervical arteries are identified well on nonregistered angiographic MIP images (Table 1). Analysis of the CNR-MIP and CNR-3D parameters showed that the angiographic contrast-tonoise ratio was on average slightly higher with image registration than without (Fig. 1a and b). However, in the ANOVA this small improvement in image quality was not significant $(p>0.05$; Table 2). ANOVA of the whole study group showed that neither image registration nor body shift had a significant influence on the quality of the carotid MRA images (Table 2). The additional subgroup ANOVA of the 14 cases with a body shift of $1 \mathrm{~mm}$ or more also showed a slight trend of image quality to improve with image registration, but this effect was also not significant $(p>0.05$; Fig. 1c and d). These results indicate firstly that standard carotid MRA needs no image registration to improve image quality. Secondly, standard breath-hold carotid MRA is generally robust against any natural body shift that may occur between acquisition of the unenhanced and the contrast-enhanced MRA sequences.

\section{Illustrative case}

Figure 2 shows an illustrative case with a body shift of $2.4 \mathrm{~mm}$ (shift class 3 ). The image quality parameters are given in Table 3. Although this body shift is relatively large, the image quality of the angiographic MIP image is high even without image registration. Automated image registration was then applied, similar to standard pixelshifting that is routinely used in DSA. However, such image registration resulted in nearly identical images and did not improve image quality much further. These results indicate that nonregistered carotid MRA was robust against

Table 1 Image quality parameters with and without image registration for the whole study group

\begin{tabular}{|c|c|c|c|c|c|c|c|}
\hline \multirow[t]{2}{*}{ Registration method } & \multirow[t]{2}{*}{ Sample size } & \multicolumn{2}{|c|}{ VDP (\%) } & \multicolumn{2}{|c|}{ CNR-MIP } & \multicolumn{2}{|c|}{ CNR-3D } \\
\hline & & Mean & (SD) & Mean & (SD) & Mean & (SD) \\
\hline None & 370 & 99.9 & $(0.8)$ & 7.02 & $(1.57)$ & 13.37 & (3.09) \\
\hline Linear & 370 & 99.9 & $(0.8)$ & 7.05 & $(1.58)$ & 13.44 & (3.14) \\
\hline Affine & 370 & 99.9 & $(0.8)$ & 7.05 & $(1.58)$ & 13.46 & (3.14) \\
\hline Warp & 370 & 99.9 & $(0.8)$ & 7.06 & $(1.58)$ & 13.52 & $(3.16)$ \\
\hline
\end{tabular}

Comparison of image registration methods by ANOVA is summarized in Table 2

$V D P$ vessel detection probability, $C N R-M I P$ contrast-to-noise ratio in MIP images, $C N R-3 D$ contrast-to-noise ratio in $3 \mathrm{D}$ subtraction image volumes, $S D$ standard deviation 
Fig. 1 Comparison of image registration methods: 1 no image registration, 2 linear, 3 affine and 4 warp registration. The ANOVA of all 370 MRAs showed a slight trend of CNRMIP (a) and CNR-3D (b) to improve with image registration, but this effect was not significant $(p>0.05)$. An additional subgroup ANOVA of the 14 cases with body shift $\geq 1 \mathrm{~mm}$ showed a similar trend for CNRMIP (c) and CNR-3D (d) that was also not significant $(p>0.05)$

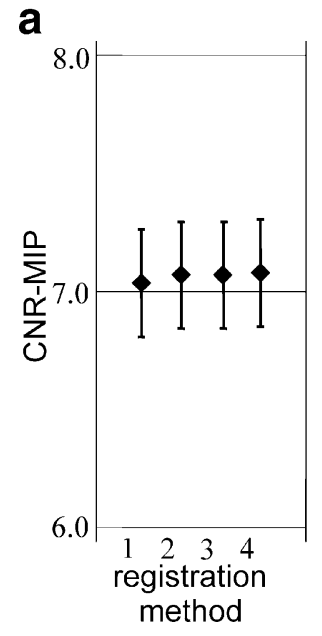

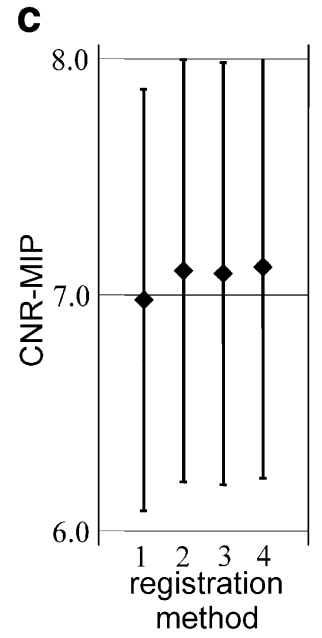

body shift in this case. Additional information is given in the legend of Fig. 2.

\section{Discussion}

Carotid artery stenosis is a treatable risk factor for ischaemic stroke [1-4]. Current guidelines recommend endarterectomy or stent-protected percutaneous transluminal angioplasty as treatments for symptomatic severe carotid stenosis with $70-99 \%$ reduction in luminal diameter, as defined by the North American Symptomatic Carotid Endarterectomy Trial (NASCET) [1]. Symptomatic moderate degree carotid stenoses in the $50-69 \%$ range may equally require treatment [1]. In addition, in selected patients with asymptomatic $60-99 \%$ carotid stenosis, endarterectomy may also be considered as a primary preventative measure according to current guidelines [2]. Quantitative assessment of carotid stenosis is thus an essential prerequisite for therapeutic decision-making in individual cases [18]. Such grading requires adequate angiographic image quality.

DSA is the current gold standard in carotid artery imaging since it combines high image contrast with high spatial resolution. However, catheter-based DSA bears the risk of complications due to its invasiveness, specifically the risk of stroke. Noninvasive diagnostic alternatives include duplex ultrasonography, CTA and MRA [5, 19].

Table 2 Comparison of image registration methods by two-factor ANOVA

\begin{tabular}{|c|c|c|c|c|c|}
\hline Image quality parameter & Source of variation & Degrees of freedom & Mean square & $F$ ratio $^{\mathrm{a}}$ & $P$ value \\
\hline \multirow[t]{3}{*}{ VDP } & Registration method & 3 & 0.01 & 0.01 & 0.999 \\
\hline & Body shift class & 2 & 0.65 & 1.08 & 0.338 \\
\hline & Residual & 1,474 & 0.60 & & \\
\hline \multirow[t]{3}{*}{ CNR-MIP } & Registration method & 3 & 0.13 & 0.05 & 0.984 \\
\hline & Body shift class & 2 & 2.87 & 1.15 & 0.317 \\
\hline & Residual & 1,474 & 2.49 & & \\
\hline \multirow[t]{3}{*}{ CNR-3D } & Registration method & 3 & 1.48 & 0.15 & 0.929 \\
\hline & Body shift class & 2 & 22.82 & 2.33 & 0.098 \\
\hline & Residual & 1,474 & 9.81 & & \\
\hline
\end{tabular}

ANOVA (analysis of variance) of the three image quality parameters with the two independent factors "image registration method" and "body shift class" revealed no statistically significant results at $p<0.05$, i.e. neither image registration nor body shift had a significant influence on the MRA image quality. A comparison of registration methods is given in Fig. 1

$V D P$ vessel detection probability, $C N R-M I P$ contrast-to-noise ratio in MIP images, $C N R-3 D$ contrast-to-noise ratio in $3 \mathrm{D}$ subtraction image volumes

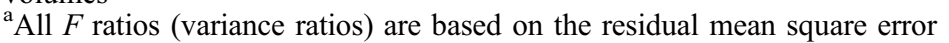



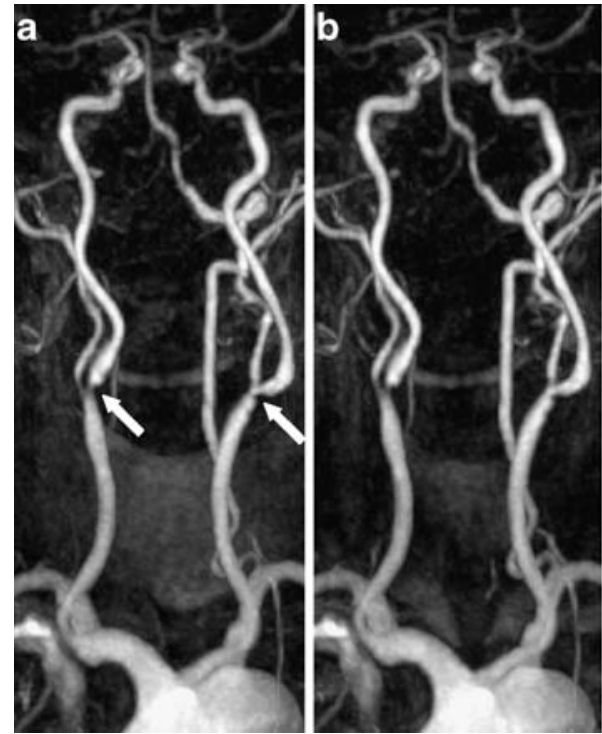

Fig. 2 Illustrative case. A 73-year-old woman was admitted for coronary artery bypass surgery. Duplex ultrasonography in the preoperative workup revealed bilateral carotid stenoses that were further evaluated by using contrast-enhanced MRA with the subtraction technique. Coronal maximum intensity projection (MIP) images are shown without image registration (a) and with linear (b), affine (c) and warp (d) registration. The arteries are depicted well, although there was an estimated body shift of $2.4 \mathrm{~mm}$ between acquisition of the unenhanced and the subsequent contrastenhanced MRA sequences. The registered images (b-d) looked relatively similar to the nonregistered image (a), indicating no need

Duplex ultrasonography is being used as a screening method, whereas CTA and MRA provide images of the entire cervical arterial tree that may be referred to during surgical or neuroendovascular intervention $[7,19,20]$. For the detection of severe $70-99 \%$ carotid artery stenosis according to the NASCET criteria, a recent meta-analysis found contrastenhanced MRA to be more sensitive and specific than CTA or duplex ultrasonography (respective sensitivities of 94\%, 76\% and $89 \%$ with specificities of $93 \%, 94 \%$ and $84 \%$ ) [7].

Carotid CTA and MRA are competitive methods with specific advantages and disadvantages [7]. Currently, CTA with multidetector CT can provide higher spatial resolution but with less vascular contrast than MRA. Such carotid CTA is usually performed without mask subtraction, i.e. only a primarily contrast-enhanced data set is acquired. Diagnosis is

Table 3 Image quality parameters in the illustrative case

\begin{tabular}{llll}
\hline $\begin{array}{l}\text { Registration } \\
\text { method }\end{array}$ & \multicolumn{3}{l}{ Image quality parameter } \\
\cline { 2 - 4 } & VDP & CNR-MIP & CNR-3D \\
\hline None & 100 & 6.54 & 11.86 \\
Linear & 100 & 6.68 & 12.25 \\
Affine & 100 & 6.77 & 12.43 \\
Warp & 100 & 6.86 & 12.49 \\
\hline
\end{tabular}
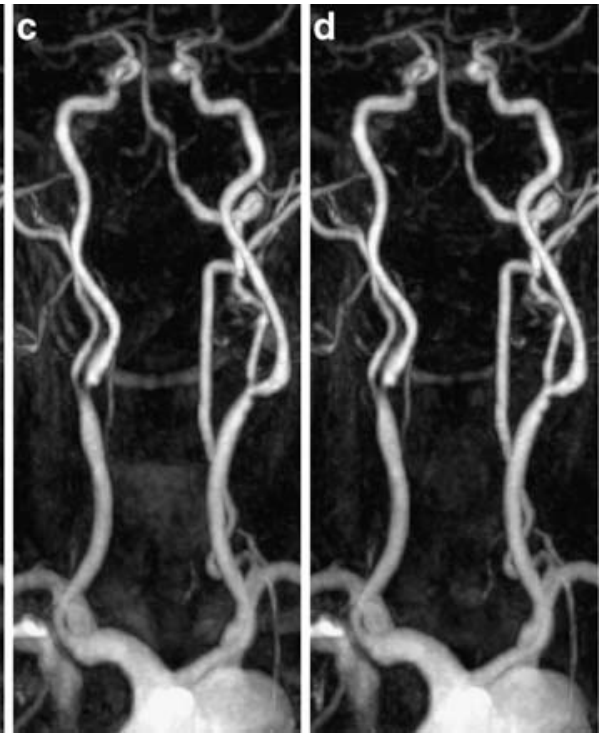

for image registration in this case. There is greater than $80 \%$ stenosis at the origin of the right internal carotid artery (arrow), greater than $60 \%$ stenosis at the origin of the left internal carotid artery (arrow) and an occlusion of the right vertebral artery. The carotid stenoses and the unilateral vertebral occlusion were asymptomatic. In addition to the intended coronary bypass grafting, the more severe right-sided carotid stenosis was treated with endarterectomy and patch angioplasty for stroke prevention. The patient has had no signs or symptoms of cerebral ischaemia on the current follow-up interval of 3 years

then based on the resulting three-dimensional image volume. Multiplanar reformation allows a detailed analysis of individual arteries, particularly the quantification of luminal narrowing. However, without mask subtraction it is difficult to generate DSA-like images since bony anatomy and calcified plaque components frequently overlie the cervical arteries in radiographic projections, even after manual image segmentation [8, 9, 21]. Such DSA-like images may, however, still be useful to attending clinicians who are used to interpreting DSA images.

In this context it is noteworthy that CTA can also be used with mask subtraction whereby an unenhanced CTA data set is subtracted from a subsequently acquired contrastenhanced counterpart $[8-10,22]$. However, subtraction artefacts due to slight body shifts may occur between scans in such examinations [22]. It has been shown that these artefacts may be eliminated and overall image quality of carotid subtraction CTA improved by image registration [8-10]. Furthermore, considering MRA in peripheral arterial disease, it has been shown that automated image registration may improve angiographic image quality significantly, specifically in the lower leg and in circumstances when a patient has shifted their legs by $1 \mathrm{~mm}$ or more between acquisition of the unenhanced and the contrast-enhanced MR sequences. This led to the question whether the image quality of contrast-enhanced carotid 
MRA might equally be improved by image registration. However, the present study did not find a similar effect of image registration in carotid MRA within the observed limits of natural body shift. This nonsignificant study result is, nonetheless, a positive finding for conventional carotid MRA since it confirms that carotid MRA is robust against naturally occurring body shifts. This is in contrast to subtraction CTA, where angiographic image quality was found to be decreased by such shifts that may occur between acquisition of the unenhanced and the contrastenhanced CTA sequences $[8,9]$.

The study finding may be explained in the context of neck anatomy and corresponding MRA signal intensities: the T1-weighted MRA images are dominated by signals from contrast-enhanced neck arteries as well as from surrounding fatty tissue. In contrast to the arteries in the lower leg, the cervical arteries are surrounded by less fatty soft tissue or marrow in adjacent bones that might cause subtraction artefacts in the case of body shift. In addition, naturally occurring body shift in the neck appears to be less than in the lower legs. Furthermore, in comparison to DSA and CTA, arterial calcifications and bony anatomy in the neck return little or no MR signal and thus cannot cause subtraction artefacts in carotid MRA [8-10].

In conclusion, standard contrast-enhanced carotid MRA usually requires no image registration to improve image quality and is generally robust against any naturally occurring body shift that may take place between acquisition of the unenhanced and the contrast-enhanced MRA sequences.

Open Access This article is distributed under the terms of the Creative Commons Attribution Noncommercial License which permits any noncommercial use, distribution, and reproduction in any medium, provided the original author(s) and source are credited.

\section{References}

1. Sacco RL, Adams R, Albers G et al (2006) Guidelines for prevention of stroke in patients with ischemic stroke or transient ischemic attack: a statement for healthcare professionals from the American Heart Association/American Stroke Association Council on Stroke: co-sponsored by the Council on Cardiovascular Radiology and Intervention: the American Academy of Neurology affirms the value of this guideline. Circulation 113:e409-e449

2. Goldstein LB, Adams R, Alberts MJ et al (2006) Primary prevention of ischemic stroke: a guideline from the American Heart Association/American Stroke Association Stroke Council: cosponsored by the Atherosclerotic Peripheral Vascular Disease Interdisciplinary Working Group; Cardiovascular Nursing Council; Clinical Cardiology Council; Nutrition, Physical Activity, and Metabolism Council; and the Quality of Care and Outcomes Research Interdisciplinary Working Group. Circulation 113:e873-e923

3. Grau AJ, Weimar C, Buggle F et al (2001) Risk factors, outcome, and treatment in subtypes of ischemic stroke: the German stroke data bank. Stroke 32:2559-2566
4. Fairhead JF, Rothwell PM (2006) Underinvestigation and undertreatment of carotid disease in elderly patients with transient ischaemic attack and stroke: comparative population based study. BMJ 333:525-527

5. Masdeu JC, Irimia P, Asenbaum S et al (2006) EFNS guideline on neuroimaging in acute stroke. Report of an EFNS task force. Eur J Neurol 13:1271-1283

6. Mitra D, Connolly D, Jenkins S et al (2006) Comparison of image quality, diagnostic confidence and interobserver variability in contrast enhanced MR angiography and 2D time of flight angiography in evaluation of carotid stenosis. Br J Radiol 79:201-207

7. Wardlaw JM, Chappell FM, Best JJ, Wartolowska K, Berry E (2006) Non-invasive imaging compared with intra-arterial angiography in the diagnosis of symptomatic carotid stenosis: a meta-analysis. Lancet 367:15031512

8. Loeckx D, Coudyzer W, Maes F et al (2007) Nonrigid registration for subtraction CT angiography applied to the carotids and cranial arteries. Acad Radiol 14:1562-1576

9. Lell MM, Ditt H, Panknin C et al (2007) Bone-subtraction CT angiography: evaluation of two different fully automated image-registration procedures for interscan motion compensation. AJNR Am J Neuroradiol 28:1362-1368
10. Loeckx D, Drisis S, Maes F, Vandermeulen D, Marchal G, Suetens P (2005) Plaque and stent artifact reduction in subtraction CT angiography using nonrigid registration and a volume penalty. Med Image Comput Comput Assist Interv Int Conf Med Image Comput Comput Assist Interv 8:361-368

11. Menke J (2008) Contrast-enhanced magnetic resonance angiography in peripheral arterial disease: improving image quality by automated image registration. Magn Reson Med 60:224-229

12. Leiner T, de Weert TT, Nijenhuis RJ et al (2001) Need for background suppression in contrast-enhanced peripheral magnetic resonance angiography. J Magn Reson Imaging 14:724-733

13. Sun Y, Parker DL (1999) Performance analysis of maximum intensity projection algorithm for display of MRA images. IEEE Trans Med Imaging 18:1154-1169

14. Woods RP, Grafton ST, Holmes CJ, Cherry SR, Mazziotta JC (1998) Automated image registration: I. General methods and intrasubject, intramodality validation. J Comput Assist Tomogr 22:139-152

15. Denton ER, Sonoda LI, Rueckert D et al (1999) Comparison and evaluation of rigid, affine, and nonrigid registration of breast MR images. J Comput Assist Tomogr 23:800-805 
16. Armitage P, Berry G (1994) Statistical methods in medical research, 3rd edn. Blackwell Science, Cambridge, pp 207-214, 237-245, 461-463

17. Altman DG, Bland JM (1996) Comparing several groups using analysis of variance. BMJ 312:1472-1473

18. U-King-Im JM, Graves MJ, Cross JJ et al (2007) Internal carotid artery stenosis: accuracy of subjective visual impression for evaluation with digital subtraction angiography and contrast-enhanced MR angiography. Radiology 244:213-222
19. Clevert DA, Johnson T, Jung EM et al (2007) Color Doppler, power Doppler and B-flow ultrasound in the assessment of ICA stenosis: Comparison with 64-MD-CT angiography. Eur Radiol 17:2149-2159

20. Qureshi AI, Alexandrov AV, Tegeler $\mathrm{CH}$, Hobson RW, Dennis Baker J Hopkins LN (2007) Guidelines for screening of extracranial carotid artery disease: a statement for healthcare professionals from the multidisciplinary practice guidelines committee of the American Society of Neuroimaging; cosponsored by the Society of Vascular and Interventional Neurology. J Neuroimaging 17:19-47
21. van Velsen EF, Niessen WJ, de Weert TT et al (2007) Evaluation of an improved technique for lumen path definition and lumen segmentation of atherosclerotic vessels in CT angiography. Eur Radiol 17:1738-1745

22. Lell MM, Ditt H, Panknin C et al (2008) Cervical CT angiography comparing routine noncontrast and a late venous scan as masks for automated bone subtraction: feasibility study and examination of the influence of patient motion on image quality. Invest Radiol 43:27-32 